\title{
Dok-3 deficient mice display different immune clustering and Tim-3 expression
}

\author{
Wenjiang Yan ${ }^{1,3+} \mathbb{0}$, Yijia Tian ${ }^{2 \dagger}$, Peng Sun ${ }^{1,4+}$, Jingjing Yang ${ }^{1}$, Na Li ${ }^{1}$, Yuanyuan Sun ${ }^{1}$, Shuangxi Wang ${ }^{1 *}$
}

and Cheng Zhang ${ }^{1 *}$

\begin{abstract}
Background: Dok-3 has been shown to play an important role in immune system. Tim-3 also has been recognized as an important immune regulator which involves in many diseases. The relationship of them is still unclear.

Methods: We detected the expression of Tim-3 on spleen immune cells from Dok-3 deficient mice and control mice by flow cytometry.

Results: In this article, we found that Dok-3 $3^{-1}$ mice display almost entirely different immune clustering characteristics compared with wild type 129 mice. The CD4 T cells and CD8 T cells decreased and DC cells, macrophages, MDSCs increased when the Dok-3 gene knocked-out. The Tim-3 expression on CD4 T cells, CD8 T cells, NK cells, DC cells increased when the Dok-3 gene knocked-out. The macrophages and MDSCs just display the opposite results.

Conclusions: Although Dok-3 $3^{-1}$ mice display different immune clustering and Tim-3 expression, the mechanism still needs further study.
\end{abstract}

Keywords: Dok-3, Immune cells proportion, Tim-3

\section{Background}

The Dok (downstream of kinase) family consists of seven members, with each possessing an NH2-terminal pleckstrin homology $(\mathrm{PH})$, a central phosphotyrosine-binding (PTB) and a C-terminal tyrosine-rich domain [1]. Dok-1, 2 and 3 are expressed in hematopoietic cells, while the other Dok members are preferentially expressed in cells of the nervous or muscular system [2]. Dok proteins function mainly as adaptors to facilitate protein-protein interactions since they have no catalytic activity [1]. Given Dok-1 to Dok-3 are also expressed in the immune cells, they play an important role in the development of the tumor progression. Recent researches showed that they have been identified as not only tumor suppressors

\footnotetext{
*Correspondence: shuangxiwang@sdu.edu.cn; zhangc@sdu.edu.cn ${ }^{+}$Wenjiang Yan, Yijia Tian and Peng Sun contributed equally to this work ${ }^{1}$ The Key Laboratory of Cardiovascular Remodeling and Function Research, Chinese Ministry of Education and Chinese Ministry of Health, and The State and Shandong Province Joint Key Laboratory of Translational Cardiovascular Medicine, Qilu Hospital of Shandong University, No. 107 Wenhua Xi Road, Jinan, Shandong, China Full list of author information is available at the end of the article
}

for lung cancer in human and mice [3] but also the depressors in the development of aggressive histiocytic sarcoma [4].

Dok-3 has been shown to play an important role in immune system. First, it can inhibit the $\mathrm{Ca}^{2+}[5]$ and JNK [6] activation in B cell receptor signaling. Secondly, macrophages are more sensitive to LPS-induced ERK activation with subsequent upregulation of a gene expression profile that promotes endotoxin tolerance in the absence of Dok-3 [7]. Finally, Dok-3 plays a critical and positive role in TLR3 signaling by enabling TRAF3/TBK1 complex formation and facilitating TBK1 and IFN regulatory factor 3 activation and the induction of IFN- $\beta$ production [8].

T-cell immunoglobulin and mucin-domain-containing molecule 3 (Tim-3) are a membrane protein initially identified as a negative regulator of Th1 immunity [9, 10]. A strong correlation between Tim-3 expression and the immune cell function has been confirmed. Recently, Tim-3 was shown to play important roles on activated Th17 [11], Tc1 [12], macrophages/monocytes [13, 14], dendritic cells (DCs) [15] and natural killer (NK) cells 
[16]. However, the relationship between the Dok-3 and Tim-3 in the immune cells is still unclear. Here, we will focus on the immune cell proportion and Tim-3 expression in the Dok-3 deficient mice.

\section{Results}

Dok-3 deficient mice display different immune cell proportions compared with 129 mice

We analyzed the CD4 T cells, CD8 T cells, NK cells, DC cells, macrophages, MDSCs in the spleens from the two mice group. As shown in Fig. 1a, the CD4 T cells marked by $\mathrm{CD}^{+} \mathrm{CD}^{+}$in $\mathrm{Dok}^{-3^{-1}}$ mice were much fewer than that from 129 mice $\left(129,38.73 \pm 2.395\right.$; Dok- $3^{-1-}$, $24.25 \pm 1.596)$. The same tendency was found in CD8 T cells marked by $\mathrm{CD}^{+}{ }^{+} \mathrm{CD}^{+}$(Fig. 1 b, $129,18.20 \pm 1.857$; Dok- $\left.3^{-1-}, 12.35 \pm 0.848\right)$. In contrast, Dok- $3^{-1-}$ mice showed much more DC cells, macrophages and MDSCs than 129 mice. As shown in Fig. 2b, DC cells marked by $\mathrm{CD}^{-} \mathrm{CD}^{-} 1 \mathrm{c}^{+}$display much higher level in Dok$3^{-1-}$ mice compared with 129 mice $(129,5.60 \pm 0.674$; Dok-3 $\left.{ }^{-1-}, 8.17 \pm 0.857\right)$. Macrophages $\left(\mathrm{CD}^{-} \mathrm{CD} 11 \mathrm{c}^{+}\right)$ from Dok- $3^{-1-}$ mice were significantly higher than that from 129 mice (Fig. 3a, 129, $12.33 \pm 2.131$; Dok- $3^{-1-}$, $31.67 \pm 3.336)$. $\mathrm{CD}^{-} \mathrm{Gr}-1^{+}$was used to mark the MDSC cells (Fig. 3b). The results show that Dok- $3^{-1-}$ mice have much more MDSCs than 129 mice $(129,9.72 \pm 1.886$; Dok- $\left.3^{-1-}, 23.73 \pm 3.150\right)$. However, the NK cells which were marked by $\mathrm{CD}^{-} \mathrm{NK} 1.1^{+}$display no difference between this two group mice (Fig. 2a). In conclusion, the Dok-3 $3^{-1-}$ mice display almost entirely different immune clustering characteristics compared with wild type 129 mice. The CD4 T cells and CD8 T cells decreased and DC cells, macrophages, MDSCs increased when the Dok-3 gene knocked-out.

\section{Dok-3 deficient mice display different Tim-3 expressions compared with 129 mice}

Since Tim-3 plays an important role in the immune cells and the Dok $-3^{-l-}$ mice display different immune cell proportions, we detected the expression of Tim-3 on these immune cells. As it is shown in Figs. 1a, b, 2a, b, the Tim-3 expression in Dok- $3^{-1-}$ mice was much higher than that in 129 mice. This results include the CD4 cells (Fig. 1a, 129, $8.45 \pm 1.568$; Dok $-3^{-/-}, 16.21 \pm 3.064$ ), CD8 cells (Fig. 1b, $129,12.68 \pm 2.950 ;$ Dok- $\left.^{-/-}, 25.20 \pm 2.595\right)$, NK cells (Fig. 2a, 129, 30.97 \pm 2.089 ; Dok- $3^{-/-}, 47.94 \pm 2.871$ ), DC cells (Fig. 2b, 129, 38.71 \pm 1.228 ; Dok-3 ${ }^{-/-}$, $49.95 \pm 3.191$ ). Although the total NK cells number displays no difference
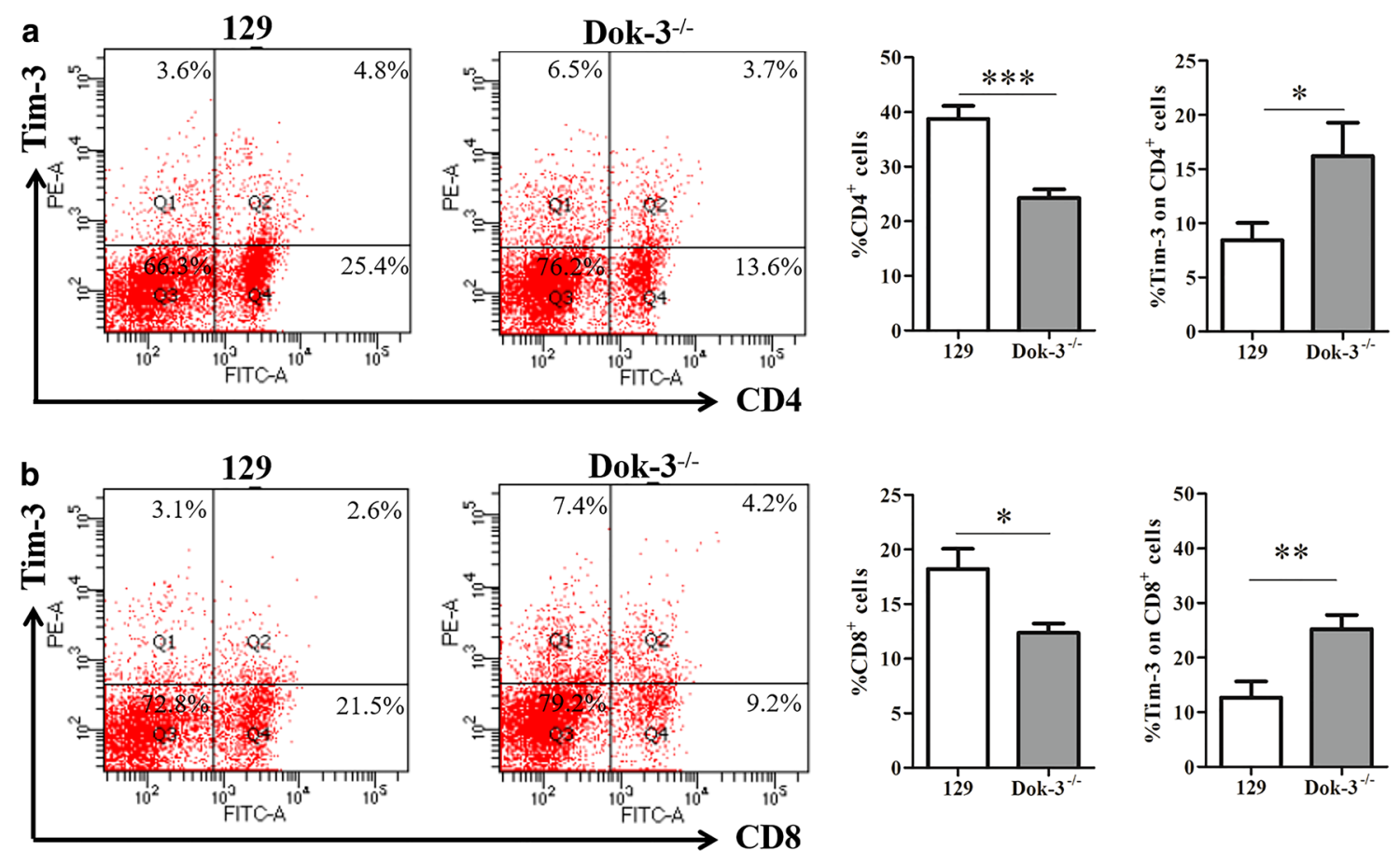

Fig. $1 \mathrm{CD}^{+} \mathrm{T}$ cells and $\mathrm{CD} 8^{+} \mathrm{T}$ cells proportion and the Tim-3 expression on these cells in two group mice. Spleen immune cells were isolated from 129 mice $(n=6)$ and Dok- $3^{-1-}$ mice $(n=6)$. a Flow cytometry $(F C M)$ analysis of Tim-3 expression on CD4 ${ }^{+}$T cells marked by CD3 ${ }^{+}$CD4 ${ }^{+}$ (left), and the statistical graph was shown (right), CD4 ${ }^{+}$T cells (129, 38.73 $\pm 2.395 ;$ Dok-3 $\left.{ }^{-/-}, 24.25 \pm 1.596\right)$ and Tim-3 expression (129, 8.45 \pm 1.568 ; Dok-3 $\left.3^{-/}, 16.21 \pm 3.064\right)$. b Flow cytometry (FCM) analysis of Tim-3 expression on CD8 ${ }^{+} \mathrm{T}$ cells marked by $\mathrm{CD} 3^{+} \mathrm{CD} 8^{+}$(left), and the statistical graph was shown (right), $C D 8^{+} T$ cells $\left(129,18.20 \pm 1.857\right.$; Dok-3 $\left.3^{-/-}, 12.35 \pm 0.848\right)$ and Tim-3 expression $\left(129,12.68 \pm 2.950 ;\right.$ Dok- $\left.3^{-/-}, 25.20 \pm 2.595\right)$. Both the $\mathrm{CD}^{+} \mathrm{T}$ cells and $\mathrm{CD} 8^{+} \mathrm{T}$ cells' gated strategy are shown in Additional file 1: Figure $\mathrm{S} 1 .{ }^{*} p<0.05,{ }^{* *} p<0.01,{ }^{* * *} p<0.001$ 

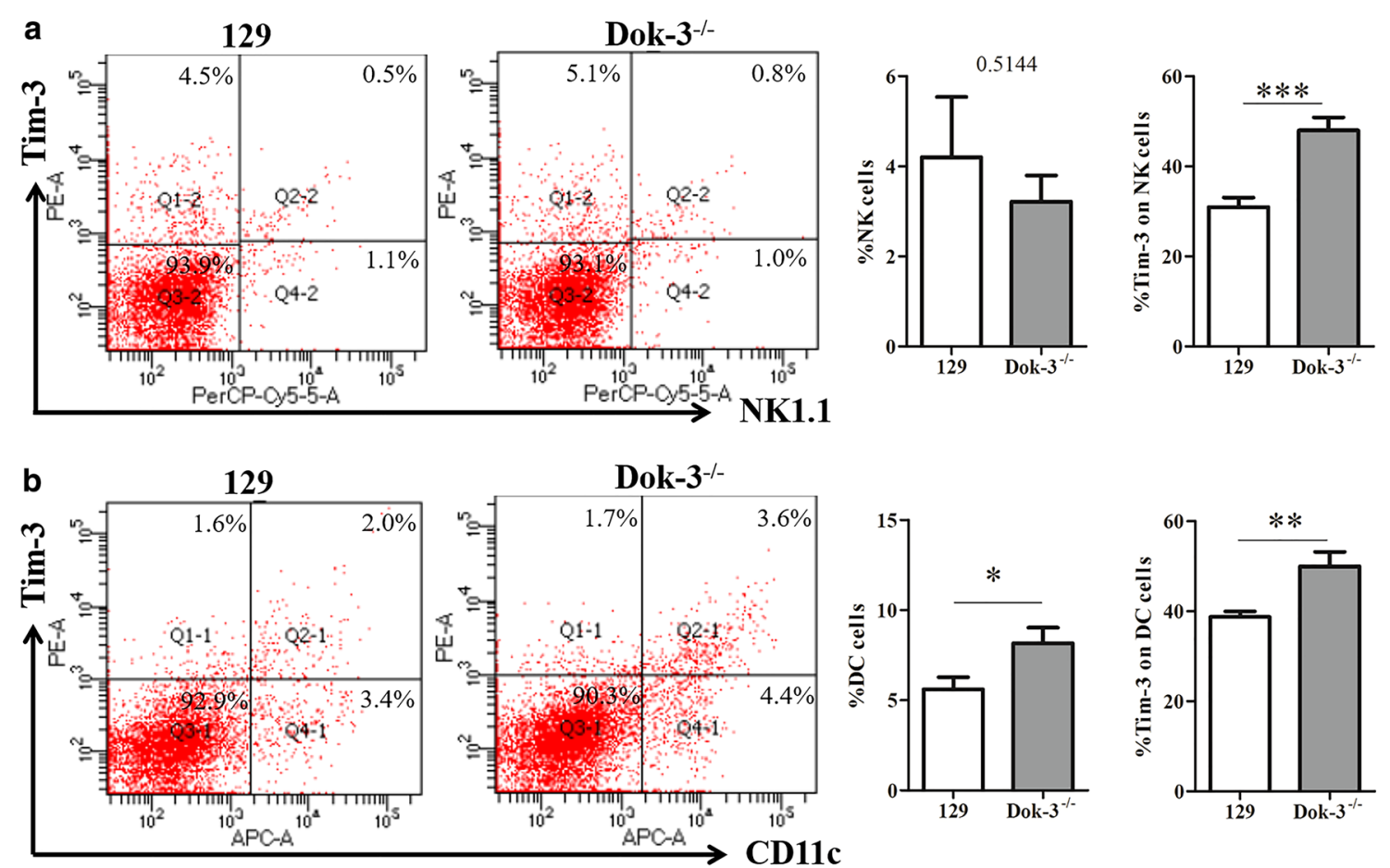

Fig. 2 NK cells and DC cells proportion and the Tim-3 expression on these cells in two group mice. Spleen immune cells were isolated from

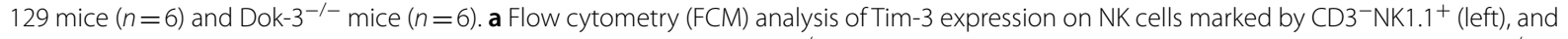
the statistical graph was shown (right), NK cells (129, 4.20 \pm 1.335 ; Dok- $\left.3^{-1-}, 3.22 \pm 0.577\right)$ and Tim-3 expression (129, 30.97 \pm 2.089 ; Dok-3 ${ }^{-1-}$, $47.94 \pm 2.871$ ). b Flow cytometry (FCM) analysis of Tim-3 expression on DC cells marked by CD3 ${ }^{-}$CD $11 \mathrm{C}^{+}$(left), and the statistical graph was shown (right), DC cells $\left(129,5.60 \pm 0.674\right.$; Dok- $\left.3^{-1-}, 8.17 \pm 0.857\right)$ and Tim-3 expression $\left(129,38.71 \pm 1.228\right.$; Dok- $\left.3^{-/-}, 49.95 \pm 3.191\right)$. Both the NK cells and DC cells' gated strategy are shown in Additional file 1: Figure S1. ${ }^{*} p<0.05,{ }^{* *} p<0.01,{ }^{* * *} p<0.001$

between this two group mice, Dok- $3^{-1-}$ mice have a much higher Tim-3 expression on NK cells than 129 mice (Fig. 2a). Contrary to the immune cells above, the macrophages $\left(129,28.61 \pm 4.120\right.$; Dok $-3^{-/-}, 16.18 \pm 1.874$ ) and MDSCs (129, $19.58 \pm 1.661 ;$ Dok- $\left.3^{-1-}, 12.58 \pm 1.315\right)$ have a much lower expression of Tim-3 in Dok- $3^{-1-}$ mice (Fig. 3). In conclusion, the Tim-3 expression on CD4 T cells, CD8 T cells, NK cells, DC cells increased when the Dok-3 gene knocked-out. The macrophages and MDSCs just display the opposite results.

\section{Methods}

\section{Mice}

129 S1/SvImJ mice (129 mice) were obtained from Huafukang (Beijing, China). DOK3-deficient 129 S1/ SvImJ mice were kindly provided by Mary Beth Humphrey from University of Oklahoma. The mice were housed in a pathogen-free room which could keep appropriate temperature and humidity. The mice were killed before the experiment. All animal experimental procedures were approved by the Shandong University Animal Care Committee and performed in accordance with the Animal Management Rules of the Chinese Ministry of Health.

\section{Spleen cell isolation and flow cytometry}

Spleen immune cells were isolated from 129 mice $(n=6)$ and Dok- $3^{-1-}$ mice $(n=6)$. Mice spleens were isolated from the body and grinded by the copper grid. The immune cells of spleen were obtained after the $10 \mathrm{~min}$ incubation with red blood cell lysis buffer. The cells were stained with anti-mouse Tim3-PE (ebioscience), anti-mouse CD3-APC (ebioscience) or anti-mouse CD3-FITC (ebioscience) which was used in DC cells marking, anti-mouse CD4-FITC (ebioscience), anti-mouse CD8-FITC (ebioscience), anti-mouse CD11b-Pe-cy-7 (ebioscience), anti-mouse NK1.1-PerCP-5.5 (ebioscience), anti-mouse CD11cAPC (ebioscience), anti-mouse Gr-1-FICT (ebioscience) for $30 \mathrm{~min}$. At least 10,000 cells were analyzed by a FACSAriaII. Cells were gated based on their forward and side scatter properties. The cells' gated strategy is shown in Additional file 1: Figure S1. 

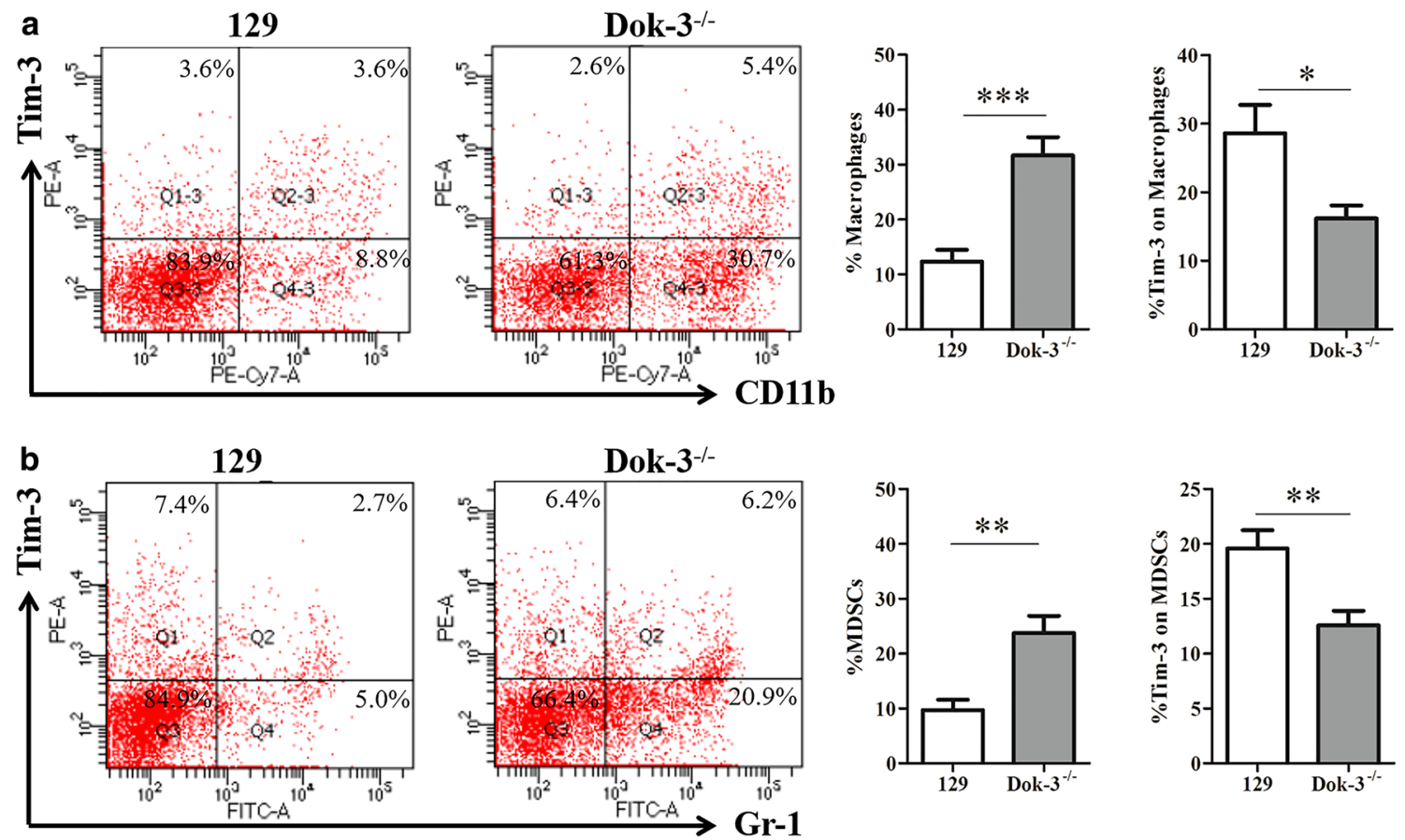

Fig. 3 Macrophages and MDSCs proportion and the Tim-3 expression on these cells in two group mice. Spleen immune cells were isolated from

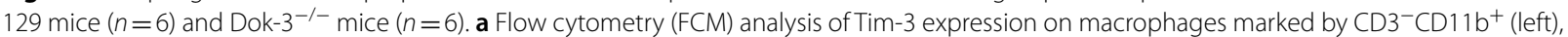
and the statistical graph was shown (right), macrophages (129, 12.33 \pm 2.131 ; Dok-3 $\left.{ }^{-1-}, 31.67 \pm 3.336\right)$ and Tim-3 expression (129, 28.61 \pm 4.120 ; Dok-3 $\left.{ }^{-/-}, 16.18 \pm 1.874\right)$. b Flow cytometry (FCM) analysis of Tim-3 expression on MDSCs marked by CD3 ${ }^{-}$CD $11 b^{+}$(left), and the statistical graph was shown (right), MDSCs (129, 9.72 \pm 1.886 ; Dok-3-/-, $23.73 \pm 3.150)$ and Tim-3 expression $\left(129,19.58 \pm 1.661\right.$; Dok-3 $3^{-/}$, 12.58 \pm 1.315$)$. Both the macrophages and MDSCs' gated strategy are shown in Additional file 1: Figure S1. ${ }^{*} p<0.05,{ }^{* *} p<0.01,{ }^{* * *} p<0.001$

\section{Statistical analyses}

All the data were analyzed by the GraphPad Prism 5 (GraphPad Software Inc., San Diego, CA). The unpaired $t$ test was used for comparison between groups. Data were reported as mean values \pm SEM. Value of $p<0.05$ was considered as significant difference.

\section{Discussion}

In this work, we present a comprehensive analysis of the immune cells proportion difference between the 129 mice and Dok- $3^{-1-}$ mice. We find that the CD4 T cells and CD8 T cells decreased and DC cells, macrophages, MDSCs increased when the Dok-3 gene knocked-out. NK cells display no difference between the two group mice.

Dok-3 has been demonstrated to be the key molecule in the immunity especially in the function of macrophages [5-8]. Here, we report the immune clustering alteration caused by Dok-3. The main reason why immune cells proportion changed is the proliferation, differentiation and apoptosis of these cells. But there is no evidence to prove that Dok-3 plays important role in the proliferation, differentiation and apoptosis of the immune cells. So, we still need more work to find the mechanism why Dok-3 affects the changed immune cells proportion.

Tim-3 has been recognized as an important immune regulator which expresses in both innate and adaptive immune cells. Increased evidence has shown that dysregulation of Tim-3 expression on peripheral CD4, CD8 $\mathrm{T}$ cells and monocytes is closely related to many diseases [11, 12, 17]. However, the roles of Dok-3 in Tim-3 expression on immune cells remain largely unknown. Here, for the first time, we demonstrated the knocked-out of Dok-3 gene which affects the Tim-3 expression on immune cells. Our data provide a previously unrecognized link between Dok-3 gene and the Tim-3 expression.

There is evidence to prove that Tim-3 is related to the apoptosis of some immune cells, especially the CD8 T cells [18]. In this study, the Dok- $3^{-1-}$ mice display the decreased CD8 T cells and increased Tim-3 expression (Fig. 1b). Maybe the high Tim-3 expression leads to the apoptosis of CD8 $\mathrm{T}$ cells which is the main reason why CD8 T cells decrease. 


\section{Conclusion}

The data presented in this study suggest that Dok-3 involves in the immune cells clustering and Tim-3 expression. But the mechanism why immune cells display this proportion and difference Tim-3 expression still needs further studies.

\section{Additional file}

Additional file 1: Figure S1. Gated strategy of Flow cytometry for the spleen cells.

\section{Abbreviations}

Dok-3: downstream of kinase 3; Tim-3: T cell immunoglobulin- and mucindomain-containing molecule-3; DC: dendritic cell; MDSC: myeloid-derived suppressor cell.

\section{Acknowledgements}

Not applicable.

\section{Authors' contributions}

WY, YT and PS designed and conducted the analysis and wrote the manuscript. JY, NL and YS collected and evaluated the data. SW and CZ contribute to the design, analysis and revised the manuscript. $C Z$ is the guarantor of the work and takes the responsibility and accuracy for the integrity of the results. All authors read and approved the final manuscript.

\section{Funding}

This study was supported by National Natural Science Foundation of China (Nos. 81500339, 81400195, 81600344), Shandong Provincial Natural Science Foundation, China (Nos. ZR2016HQ29, ZR2016HP01).

\section{Availability of data and materials}

We declare that all the data and materials in this article are available.

\section{Ethics approval and consent to participate}

The mice were killed before the experiment. All animal experimental procedures were approved by the Shandong University Animal Care Committee and performed in accordance with the Animal Management Rules of the Chinese Ministry of Health.

\section{Consent for publication}

The authors declare that they are consent to publish this article to your journal.

\section{Competing interests}

The authors declare that they have no competing interests.

\section{Author details}

${ }^{1}$ The Key Laboratory of Cardiovascular Remodeling and Function Research, Chinese Ministry of Education and Chinese Ministry of Health, and The State and Shandong Province Joint Key Laboratory of Translational Cardiovascular Medicine, Qilu Hospital of Shandong University, No. 107 Wenhua Xi Road, Jinan, Shandong, China. ${ }^{2}$ Grade 2015 , School of Basic Medical Sciences, Clinical Medicine $(5+3)$, Shandong University, Jinan, Shandong, China. ${ }^{3}$ Key Laboratory of Infection and Immunity of Shandong Province, Jinan, China. ${ }^{4}$ Department of Intervention Oncology, Shandong Cancer Hospital and Institute, Shandong Academy of Medical Sciences, Jinan, Shandong, China.
Received: 13 July 2018 Accepted: 15 July 2019

Published online: 27 July 2019

\section{References}

1. Mashima R, Hishida Y, Tezuka T, Yamanashi Y. The roles of Dok family adapters in immunoreceptor signaling. Immunol Rev. 2009;232(1):273-85.

2. Favre C, Gérard A, Clauzier E, Pontarotti P, Olive D, Nunès JA. DOK4 and DOK5: new Dok-related genes expressed in human T cells. Genes Immun. 2003;4(1):40-5.

3. Berger $\mathrm{AH}$, Niki M, Morotti $\mathrm{A}$, et al. Identification of DOK genes as lung tumor suppressors. Nat Genet. 2010;42(3):216-23.

4. Mashima R, Honda K, Yang Y, et al. Mice lacking Dok-1, Dok-2, and Dok-3 succumb to aggressive histiocytic sarcoma. Lab Invest. 2010;90(9):1357-64.

5. Stork B, Neumann K, Goldbeck I, et al. Subcellular localization of Grb2 by the adaptor protein Dok-3 restricts the intensity of $\mathrm{Ca} 2+$ signaling in $\mathrm{B}$ cells. EMBO J. 2007;26(4):1140-9.

6. Robson JD, Davidson D, Veillette A. Inhibition of the Jun N-terminal protein kinase pathway by SHIP-1, a lipid phosphatase that interacts with the adaptor molecule Dok-3. Mol Cell Biol. 2004;24(6):2332-43.

7. Peng Q, O'Loughlin JL, Humphrey MB. DOK3 negatively regulates LPS responses and endotoxin tolerance. PLOS ONE. 2012;7(6):e39967.

8. Kim SS, Lee KG, Chin CS, et al. DOK3 is required for IFN- $\beta$ production by enabling TRAF3/TBK1 complex formation and IRF3 activation. J Immunol. 2014;193(2):840-8.

9. Sabatos CA, Chakravarti S, Cha E, et al. Interaction of Tim-3 and Tim-3 ligand regulates $T$ helper type 1 responses and induction of peripheral tolerance. Nat Immunol. 2003:4(11):1102-10.

10. Sánchez-Fueyo A, Tian J, Picarella D, et al. Tim-3 inhibits T helper type 1-mediated auto- and alloimmune responses and promotes immunological tolerance. Nat Immunol. 2003;4(11):1093-101.

11. Liberal $R$, Grant $C R$, Holder BS, et al. The impaired immune regulation of autoimmune hepatitis is linked to a defective galectin-9/tim-3 pathway. Hepatology. 2012;56(2):677-86.

12. Wu W, Shi Y, Li S, et al. Blockade of Tim-3 signaling restores the virusspecific CD8 ${ }^{+}$T-cell response in patients with chronic hepatitis B. Eur J Immunol. 2012:42(5):1180-91.

13. Monney L, Sabatos CA, Gaglia JL, et al. Th1-specific cell surface protein Tim-3 regulates macrophage activation and severity of an autoimmune disease. Nature. 2002;415(6871):536-41.

14. Zhang Y, Ma CJ, Wang JM, et al. Tim-3 negatively regulates IL-12 expression by monocytes in HCV infection. PLOS ONE. 2011;6(5):e19664.

15. Chiba S, Baghdadi M, Akiba H, et al. Tumor-infiltrating DCs suppress nucleic acid-mediated innate immune responses through interactions between the receptor TIM-3 and the alarmin HMGB1. Nat Immunol. 2012;13(9):832-42.

16. Ndhlovu LC, Lopez-Vergès S, Barbour JD, et al. Tim-3 marks human natural killer cell maturation and suppresses cell-mediated cytotoxicity. Blood. 2012;119(16):3734-43.

17. Anderson AC. Tim-3, a negative regulator of anti-tumor immunity. Curr Opin Immunol. 2012;24(2):213-6.

18. Kang CW, Dutta A, Chang LY, et al. Apoptosis of tumor infiltrating effector TIM-3+ CD8+ T cells in colon cancer. Sci Rep. 2015;5:15659.

\section{Publisher's Note}

Springer Nature remains neutral with regard to jurisdictional claims in published maps and institutional affiliations. 